\title{
An outline of the noun morphology in Vakh- Vasyugan Khanty
}

\author{
Victoria Vorobeva ${ }^{1}$, Irina Novitskaya ${ }^{2}$ \\ Tomsk State University, Tomsk Polytechnic University ${ }^{1}$
}

\begin{abstract}
:
This research presents findings resulting from a comparative analysis of the system of nominal inflection in two dialects of the Eastern Khanty language: Vakh and Vasyugan. From the sociolinguistic perspective, the Vasyugan is hardly used by the ethnic speech community.

In the analyzed dialects, the noun inflects for number, case and possession. In the morphological structure of the word these nominal categories are represented in the following order: number marker + possession marker + case marker. The focus of the comparative analysis is on similarities and differences in the system of nominal markers in the four dialects.

The noun has 3 numbers: singular, dual and plural. Each number has two forms: absolute and dependent. Their use depends on the presence or absence of possessive markers in the word structure.

Possessive markers simultaneously encode the number of the possessed and the person and number of the possessor. Our analysis of the dialectal forms of possessive markers reveals the fact that in all Eastern dialects the forms of 1SG of the possessor and the forms of the dual and plural of the possessed tend to coincide.

The category of case includes a significant number of cases in Vakh and Vasjugan dialects. These cases are nominative, lative, locative, commutative, instrumental, obsessive, translative, ablative, and approximatively. Case markers for ablative and approximatively differ in the analyzed dialects. The Comparative case is represented not in all case paradigms of the analyzed dialects. The question whether the Distributive case exists in the Eastern dialects remains open.

All in all, the system of nominal inflection in Vakh and Vasyugan dialects is for the most part similar. Although such categories as number, possession and case are shared by all dialects, the comparative analysis of their paradigms in four dialects reveals some controversial issues regarding forms and presence of morphological markers.
\end{abstract}

Keywords: Vakh-Vasyugan Khanty, morphology, number, possession, case 